\title{
Faceted Subtopic Retrieval: Exploiting the Topic Hierarchy via a Multi-modal Framework
}

\author{
Jitao Sang ${ }^{1,2}$, Changsheng $\mathrm{Xu}^{1,2}$ \\ ${ }^{1}$ National Lab of Pattern Recognition, Institute of Automation, CAS, China \\ ${ }^{2}$ China-Singapore Institute of Digital Media, Singapore \\ Email: $\{$ jtsang, csxu\}@nlpr.ia.ac.cn
}

\begin{abstract}
The overwhelming amount of web videos posted on the social media websites make effective browsing and search a challenging task. The user-provided metadata, has been proved useful in large-scale video organization and retrieval. Search result clustering, which utilizes the associated metadata to cluster the returned results into semantic groups according to its involved subtopics, has shown its advantages. Most of the existing works on search result clustering are devoted to solving the ambiguous problem resulted from general queries. In this paper, we propose the problem of faceted subtopic retrieval, which focus on more complex queries concerning political and social events or issues. Hierarchical topic model (hLDA) is adapted to exploit the intrinsic topic hierarchy inside the retrieved collections. Furthermore, this paper offers a new perspective of multi-modal video analysis by exploring the pairwise visual cues deriving from duplicate detection for constrained topic modeling. We modify the standard hierarchical topic model by integrating: 1) query related Supervision knowledge (ShLDA) and 2) duplicate Relation constraints (RShLDA). Carefully designed experiments on web-scale video dataset validate the proposed method.
\end{abstract}

Index Terms-subtopic retrieval, multi-modal analysis, search result clustering, hierarchical topic model, social media, connected multimedia

\section{INTRODUCTION}

With the development of multimedia technology and increasing proliferation of social media in Web 2.0, an overwhelming volume of images and videos have been posted to the media sharing websites, making effective browsing and searching a challenging task. It becomes extremely difficult for users to find the information they need. The quality of search engines are often far from satisfactory due to various reasons. Firstly, although ranked lists are still popular ways for organizing the search results, it is highly inefficient since users have to painstakingly browse through the long list to judge whether the results match their requirements. Secondly, a majority of the queries tend to be short, non-specific and imprecise, which makes the relevance-based rank results even unreliable.

One approach to address the above issues is search result clustering, which clusters and visualizes the search results into semantically consistent groups. The main advantage is that it favors systematic exploration and helps users get a quick overview of search results, which
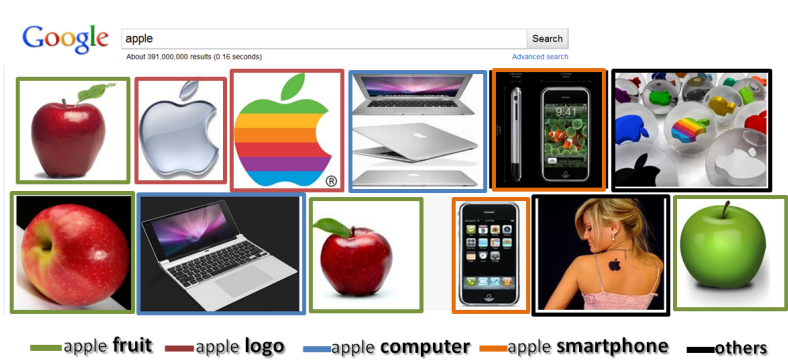

(a)

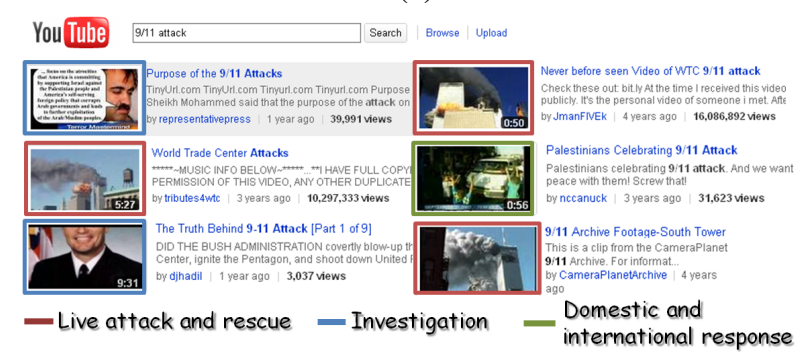

(b)

Figure 1. Example queries. (a) Ambiguous query and subtopics in its search results from Google's image search (b) Faceted query and subtopics from Youtube

decreases the effort required to locate relevant information. Moreover, when the queries are short, it is usually involved with multiple aspects of potential interest and the relevance criterion alone is not sufficient, which aggravate the limitation of the rank principle.

In search result clustering, ideally, one cluster corresponds to one subtopic of the query-related topic. Actually, according to different queries, there are two categories of subtopics. The first is called ambiguous subtopic, where the queries have multiple interpretations and each subtopic corresponds to one independent interpretation (see an example in Fig.1(a)). The ambiguous queries most involve general objects or names. Most of the previous search result clustering methods [1]-[5] are devoted to solving this ambiguous problem. Another is called faceted subtopic, where the queries have unique interpretation with each subtopic covering different facet of the query (see an example in Fig.1(b)). Most of the queries concerning political and social events or issues belong to this category, which is the focus of this work. In this paper we define the problem of faceted subtopic retrieval as 
clustering and visualizing the search results into faceted subtopics, and we present a novel multi-modal framework combining the user-provided textual metadata and the video visual content. From the perspective of utilizing the user-provided metadata, this work can be viewed as one implementation of the new research field - connected multimedia, which is devoted to bridging the semantic gap in multimedia analysis by the aid of user involvement and cultural constraints [6].

We observe that there exists an intrinsic hierarchical structure in the search results collection returned from faceted queries. Simply taking a glance at the example in Fig.1(b), we find that almost all the returned videos contain words like '9/11', 'attack', 'terrorism', 'WTC', etc. This is reasonable and implies that although diverse topics are involved in the retrieved video collection, they usually share one common topic referred in query. Each retrieved video can be viewed as a combination of the shared topic ("9/11 terrorist attack") and one subtopic (which can be enumerated as "Live attack and rescue video", "Domestic and international response afterwards", "Investigation" and "The Else: long-term effect and memorial"). Delighted from this, we extend the hierarchical Latent Dirichlet Allocation (hLDA, [7]) to exploit a two-level topic tree in the retrieved video collection and cluster the collected videos into the leaf-level subtopics.

Furthermore, we provide a novel multi-modal framework to fuse textual features (user-provided metadata, e.g., title, description, tags) and visual features (video content). Although many efforts have been targeted at building concept detector from low-level visual features, the performance of high-level detector is far from satisfactory due to the notorious semantic gap. These years nearduplicate cluster detection has attracted extensive research attentions. Several effective methods have been proposed and the detection precision is acceptable. However, typically the near-duplicate detection is based on keyframe extraction and matching from the low-level visual features. The video clips in the same near-duplicate cluster are basically derived from the same original copy and are not sufficient to represent the semantic subtopics. Instead of directly constructing the clusters from the duplicate detection, in this paper we assume that, if two videos are near-duplicate, they should be semantically clustered together and belong to the same subtopic. We propose to employ the video content for near-duplicate detection and integrate the obtained duplicate correlation between every two videos as the pairwise visual constraints on top of textual metadata based topic modeling. To this end, we extend the traditional hierarchical topic model to its relational version.

The main contributions of this paper are summarized as two aspects.

- We propose the problem of faceted subtopic retrieval and adapt the method of hierarchical topic model to exploit the latent hierarchical structure. Furthermore, query is incorporated to guide the topic hierarchy discovery and a supervised extension (ShLDA) to the standard hierarchical topic model is introduced.

- A novel multi-modal framework is presented. The pairwise duplicate relations are utilized as the visual constraints onto the textual feature based topic modeling. We further extend the ShLDA to its relational version, relational ShLDA (RShLDA).

The rest of the paper is structured as follows. In section II, we review related work on search result clustering and multi-modal video analysis. The overview of our framework is described in section III, whereas the details are presented in section IV and section V. Section VI reports our experimental results on web video dataset and section VII concludes our work.

\section{RELATED WORK}

In this section, we review the previous work on search result clustering and multi-modal video analysis. Their relations with our work are also discussed.

\section{A. Search Result Clustering}

Search result clustering, which clusters the raw returned documents into different semantic groups has been investigated in text retrieval [1], [2], image retrieval [3], [4] and video retrieval [5]. Comparing with the conventional rank list, the labeled clusters allow better topic understanding and favor systematic exploration of the search results.

Most of the previous search result clustering methods are devoted to solving the ambiguous problem resulted from non-specific queries. The queries most involve general objects or names, and the cluster labels (ambiguous subtopics) correspond to alternative interpretations of the query. For example, query 'apple' with interpretation of computer, ipod, logo and fruit [3]; query 'sting' with interpretation of musician, wrestler and film [5]. In this paper we focus on more complex queries concerning political and social events or issues. These queries have unique interpretation and the subtopics are diverse facets of the query-corresponding events (e.g. query of ' $9 / 11$ attack') or different viewpoints on controversial issues (e.g. query of 'abortion' with opposing viewpoints of 'pro-life' and 'pro-choice'). The existing methods are not adaptable to the problem of faceted subtopic retrieval as they take no consideration on the intrinsic hierarchical topic structure.

Note that the term of 'faceted retrieval' was also defined in [8]. However, their work is devoted to the TREC diversity task which aims to return a ranked list providing as much coverage for a query as possible [9]. In their case, "faceted" indicates each document may cover multiple facets of a topic.

\section{B. Multi-modal Video Analysis}

Web videos carry rich textual metadata as well as video content, which together provide important clues for video analysis and topic mining. The method of combining multiple modality features, however, is not trivial. One straight approach is to concatenate features from each 


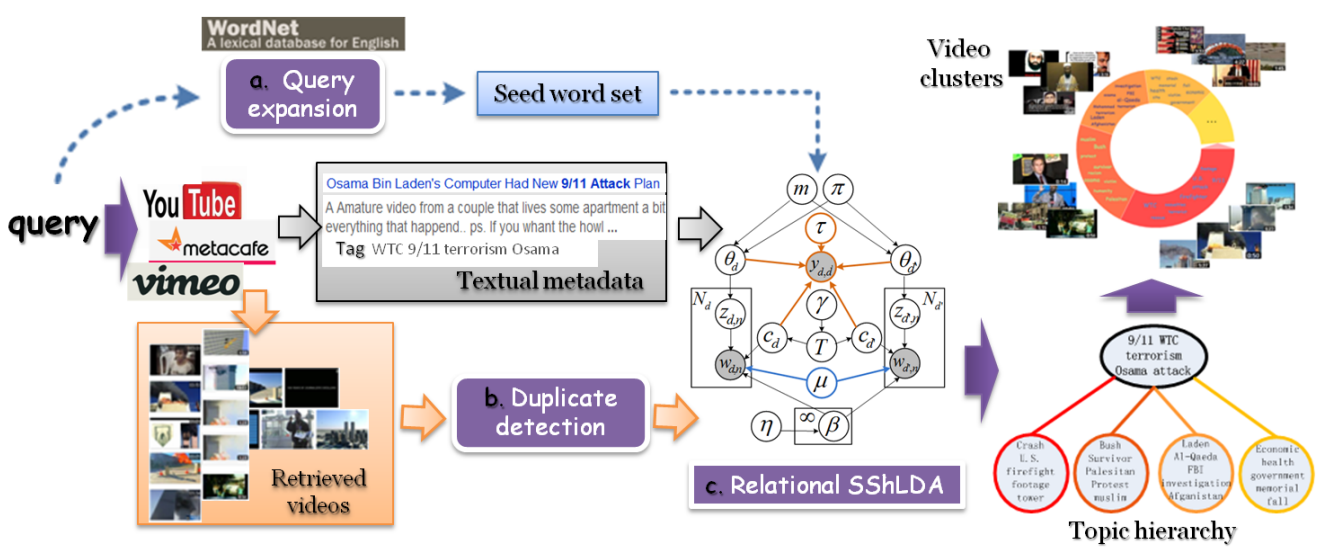

Figure 2. The proposed framework.

modality to make a long feature vector. This method will suffer from the "curse of dimensionality" [10] and concatenating the multidimensional features simply does not scale up. Another approach is to build individual models for each modality, and combine them for final decision [11], [12]. This approach lacks solid theories and the unsatisfactory performance of the visual concept detector make the learnt models unreliable and hardly generalizable. Recently, machine learning algorithms of graph-based [13], co-clustering [14] and multiple kernel learning [15] are proposed to combine multiple types of heterogeneous data. However, the expensive cost of feature processing is not acceptable for the problem of search result clustering.

In this paper, we employ the visual video content by duplicate detection and integrate the duplicate link into the topic modeling process. Effective duplicate detection methods have been proposed and the precision is satisfactory. A statistic found that there are 20\%-30\% of near duplicate videos in the web collections [16], which indicates that there are abundant near duplicate link available for topic mining in web videos.

\section{FRAMEWORK}

In this paper, we propose a relational hierarchical topic model based multi-modal framework for faceted subtopic retrieval. This work attempts to organize the search results from web video search engines into semantic clusters, with each cluster corresponding to one subtopic of the faceted query. The framework is shown in Fig.2. The input of our algorithm is the submitted queries, the web videos and associated textual metadata collected from social media websites (e.g., Youtube, Metacafe, Vimeo, etc.). The output is the generated video clusters as well as the topic hierarchy. The framework contains three components, query expansion, duplicate detection and hierarchical topic model based topic hierarchy discovery. For query expansion, we employ association mining as well as WordNet conceptual relation to expand the query words, resulting in a seed word set. The seed word set is viewed as the supervision information (we refer it as query-root-topic knowledge) for the latent root topic and will later be integrated into the topic hierarchy discovery process. The retrieved videos are sent to the duplicate detection module to find the duplicate clusters, which are then utilized as the visual constraint to guide the inference of the topic hierarchy. We note that the duplicate detection can be performed offline at the time the videos are uploaded. The details of query expansion and duplicate detection are presented in section IV. The extension to the traditional hierarchical topic model, RShLDA, is the core of our framework. RShLDA utilizes the supervision information of query-root-topic and the observed documentlevel duplicate link to help discover the topic hierarchy. After probabilistic inference, each video is assigned a single path from the root node to a leaf node. The videos assigned to the same path will be grouped together to form a semantic cluster and the subtopics in the leaf node constitute the description for the corresponding video clusters.

\section{TOPIC HIERARCHY DISCOVERY VIA HIERARCHICAL TOPIC MODELLING}

We begin this section by briefly reviewing LDA and the standard hLDA. Then we introduce our extensions to hLDA, ShLDA and RShLDA. The inference algorithms are also discussed. In the following, we will describe the models using the original terms "documents" and "words" as used in the topic model literatures (in our case, each video corresponds to one document).

\section{A. $L D A$ and $h L D A$}

Suppose we have a corpus of $M$ documents, $\left\{\mathbf{w}_{1}, \mathbf{w}_{2}, \cdots, \mathbf{w}_{M}\right\}$ containing words from a vocabulary of $V$ terms. Further we assume that the order of words in a particular document is ignored. This is a 'bag-of-words' model.

LDA: Latent Dirichlet Allocation model [17] assumes that documents are generated from a set of $K$ ( $K$ needs to be predefined) latent topics. In a document, each word $w_{i}$ is associated with a hidden variable $z_{i} \in\{1, \cdots, K\}$ indicating the topic from which $w_{i}$ was generated. The probability of word $w_{i}$ is expressed as

$$
P\left(w_{i}\right)=\sum_{j=1}^{K} P\left(w_{i} \mid z_{i}=j\right) P\left(z_{i}=j\right)
$$




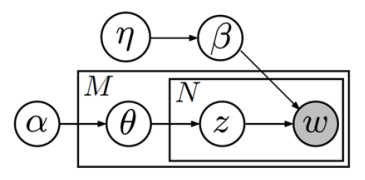

(a)

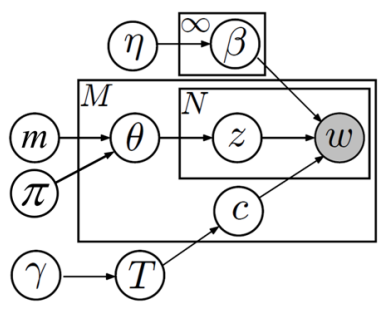

(b)

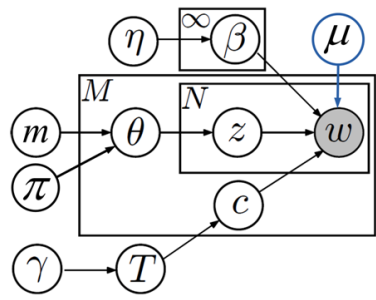

(c)

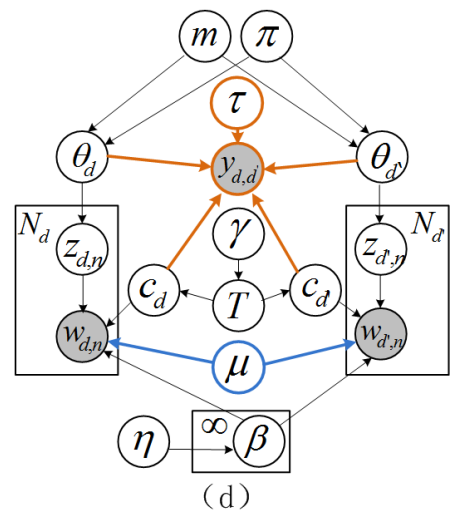

Figure 3. The graphical representation for the topic models: (a) LDA (b) hLDA (c) ShLDA and (d) RShLDA. The parameter $\mu$ controls the strength of query-root-topic supervision. The variable $y_{d, d^{\prime}}$ indicates whether the two videos are near-duplicates. The proposed ShLDA and RShLDA differs from the standard hLDA in the way the words $\mathbf{w}$ are generated.

where $P\left(w_{i} \mid z_{i}=j\right)=\beta_{i j}$ is a probability of word $w_{i}$ in topic $j$ and $P\left(z_{i}=j\right)=\theta_{j}$ is a document specific mixing weight indicating the proportion of topic $j$. LDA treats the multinomial parameters $\beta$ and $\theta$ as latent random variables sampled from a Dirichlet prior with hyperparameters $\alpha$ and $\eta$ respectively. The corresponding graphical model is shown in Fig.3(a).

hLDA: The LDA model described above has a flat topic structure. Each document is a superposition of all $K$ topics with document specific mixture weights. The hierarchical LDA model organizes topics in a tree of fixed depth $L$. Each node in the tree has an associated topic and each document is assumed to be generated by topics on a single path from the root to a leaf through the tree. Note that all documents share the topic associated with the root node, this feature of hLDA is consistent with the characteristics of search result collection we mentioned in Section I. The merit of the hLDA model is that both the topics and the structure of the tree are learnt from the training data. This is achieved by placing a nested Chinese restaurant process (nCRP) [18] prior on the tree structure. nCRP specifies a distribution on partitions of documents into paths in a fixed depth $L$-level tree. To generate a tree structure from nCRP, assignments of documents to paths are sampled sequentially, where the first document forms an initial $L$-level path, i.e. a tree with a single branch. The probability of creating novel branches is controlled by parameter $\gamma$, where smaller values of $\gamma$ result in a tree with fewer branches. In hLDA, each document is assumed drawn from the following process:

i. Pick a $L$-level path $\mathbf{c}_{d}$ from the nCRP prior: $\mathbf{c}_{d} \sim$ $n C R P(\gamma)$.

ii. Sample $L$-dimensional topic proportion vector $\theta_{d} \sim$ $\operatorname{GEM}(m, \pi)$.

iii. For each word $w_{d, n} \in \mathbf{w}_{d}$ :

(a) Choose level $z_{d, n} \sim \operatorname{Discrete}\left(\theta_{d}\right)$;

(b) Sample a word $w_{d, n} \sim \operatorname{Discrete}\left(\beta_{\mathbf{c}_{d}} \mid z_{d, n}\right)$, which is parameterized by the topic in level $z_{d, n}$ on the path $\mathbf{c}_{d}$.

The corresponding graphical model is shown in Fig.3(b).
$\mathcal{T}$ denotes the tree structure generated from the nCRP process, $\mathbf{c}$ is the selected path of documents, hyperparameter $\eta$ is the same with that in LDA and control the smoothing/sparsity of topic-word distribution ${ }^{1}$, while GEM parameters $\{m, \pi\}$ reflect the stick-breaking distribution and control the document-topic allocation. Further details of hLDA can be found in [7]. When we utilize hierarchical topic model for the video clustering task, one subtopic corresponds to one cluster. The cluster membership of each video is decided by its posterior path assignment $\mathbf{c}_{d}$. The cluster videos are sorted by their proportion on the subtopic as computed by:

$$
\frac{\sum_{w_{d, n} \in \mathbf{w}_{d}}\left|z_{d, n}=2\right|}{N_{d}}
$$

where $|\cdot|$ is indicator function and the numerator accumulates the number of words allocated at the leaf level, $N_{d}$ denotes the word number.

\section{B. $S h L D A$}

To incorporate the query-root-topic knowledge into the hierarchical topic modeling, we propose the supervised hLDA model (ShLDA). The supervision information we add is the seed word set derived from query expansion, $\mathcal{S}$. We jointly model the documents and the seed word set, in order to guide the discovery of topic hierarchy so that the words in the seed word set will have high probability in the root topic and low probability in subtopics.

We first explain how query-root-topic knowledge can be incorporated into the topic modeling process. In the standard hLDA, the topic level allocation $z_{d, n}$ for word $n$ in document $d$ is a latent variable and needs to be inferred through the model learning process. Assume we have the supervised information of $z_{d, n}$, i.e. the topic level allocation for a given word in a given document. We denote it as hard constraint when the seed set words are restricted to be shown only in the root topic. In practical applications, each word tends to be generated from every topic with different probabilities. Therefore, we relax the strong assumption that seed words only be generated from

\footnotetext{
${ }^{1} \infty$ denotes that there no constraint for the number of topics.
} 
the root topic. Instead of providing topic level allocation $z_{d, n}$ for each seed word, we modify the generative process of standard hLDA so that sampling seed words from root topic and subtopics will have different probabilities.

Specifically, the proposed ShLDA differs from hLDA in the way $w_{d, n}$ is generated. The generative process of ShLDA is:

i. Pick a $L$-level path $\mathbf{c}_{d}$ from the nCRP prior: $\mathbf{c}_{d} \sim$ $n C R P(\gamma)$.

ii. Sample $L$-dimensional topic proportion vector $\theta_{d} \sim$ $\operatorname{GEM}(m, \pi)$.

iii. For each word $w_{d, n} \in \mathbf{w}_{d}$ :

(a) Choose level $z_{d, n} \sim \operatorname{Discrete}\left(\theta_{d}\right)$;

(b) Sample a word $w_{d, n} \sim \operatorname{Constraint}\left(\mu, z_{d, n}\right)$. $\operatorname{Discrete}\left(\beta_{\mathbf{c}_{d}} \mid z_{d, n}\right)$

The corresponding graphical model is shown in Fig.3(c). Constraint $\left(\mu, z_{d, n}\right)$ is the soft constraint function defined as follows:

Constraint $\left(\mu, z_{d, n}\right)= \begin{cases}\mu \delta\left(w_{d, n} \in \mathcal{S}\right)+1-\mu, & z_{d, n}=1 \\ \mu \delta\left(w_{d, n} \notin \mathcal{S}\right)+1-\mu, & \text { else. }\end{cases}$

where $\mu(0 \leq \mu \leq 1)$ is the strength parameter of the supervision and $\delta(\cdot)$ is an indicator function with value 1 if the condition is satisfied and 0 otherwise. $\mu=0$ reduces to standard hLDA and $\mu=1$ recovers the hard constraint.

The formulation in Eq.3 provides us a flexible way to insert a prior domain knowledge into the inference of latent topics with different definitions of the constraint function, e.g. with prior information on the latent subtopics, $\mathcal{S}$ can be set independently for the specific subtopic.

\section{RShLDA}

Besides the observed words, the links between documents are also important cues for topic modeling. For each pair of documents $d$ and $d^{\prime}$, the results of duplicate detection are viewed as the observed link and modeled as a binary random variable $y_{d, d^{\prime}}$ conditioned on their contents. Duplicate link variable $y_{d, d^{\prime}}=1$ if $d$ and $d^{\prime}$ are near-duplicate, and $y_{d, d^{\prime}}=0$ otherwise. In this sense, we propose RShLDA, a relational extension to the proposed ShLDA. RShLDA is a model of data composed of documents and links between them. It embeds these data in a latent topic space which explains both the words of the documents and how they are connected.

In RShLDA, each document is first generated as in ShLDA. The duplicate links between every two documents are then modeled as binary variables. It assumes that a set of observed documents $w_{1: D, 1: N_{d}}$ and binary duplicate links between them $y_{1: D, 1: D}$ are generated by the following process ${ }^{2}$ :

\footnotetext{
2 For $w_{1: D, 1: N_{d}}, 1: D$ and $1: N_{d}$ denote the range of its subscripts, where $D$ is the number of documents and $N_{d}$ is the number of words in document $d$. Similar representation is used in $y_{1: D, 1: D \text {. }}$
}

i. For each document $d$ : performs the same generative process as ShLDA.

ii. For each pair of documents $d, d^{\prime}$ :

(a) Draw binary duplicate link indicator

$$
y_{d, d^{\prime}} \sim \psi\left(\cdot \mid \mathbf{c}_{d}, \mathbf{c}_{d^{\prime}}, \theta_{d}, \theta_{d^{\prime}}, \tau\right)
$$

The function $\psi(\cdot)$ is the link probability function that defines a distribution over the duplicate link between two documents. It is dependent on the path assignments $\mathbf{c}_{d}$, $\mathbf{c}_{d^{\prime}}$ and the topic proportions $\theta_{d}, \theta_{d^{\prime}}$ :

$$
\psi\left(y_{d, d^{\prime}}=1\right)= \begin{cases}\sigma\left(\tau^{T}\left(\theta_{d} \circ \theta_{d^{\prime}}\right)\right), & \text { if } \mathbf{c}_{d}=\mathbf{c}_{d^{\prime}} \\ 0, & \text { else. }\end{cases}
$$

where $\tau$ is coefficient parameter, $\circ$ denotes the elementwise product and $\sigma(\cdot)$ is the sigmoid function. This formulation ensures that the same latent topic assignments used to generate the content of the documents also generate the link structure. We can see that only when two documents , are assigned to the same path (subtopic), the binary variable has the probability to be one. The more similar the topic proportions of the two documents are, the more likely the duplicate link exists. This is consistent with our assumption in section I. Fig.3(d) illustrates the graphical model for RShLDA for a single pair of documents. Note that the full model contains $D^{2}$ duplicate link variables and is difficult to illustrate.

\section{Inference}

The central computational problem in Bayesian topic modeling is the posterior inference, which inverts the generative process to estimate the conditional distribution of the latent variables in the model. In the hierarchical topic model, these latent variables provide the tree structure and node parameters. As exact inference is intractable, we resort to Gibbs sampling for approximate inference.

We modify the Gibbs sampling method in hLDA to estimate the posterior for RShLDA. The core idea of Gibbs sampling is to sample from a Markov chain whose stationary distribution is the posterior. Each latent variable is iteratively sampled conditioned on the observations and all the other latent variable. Following [7]'s approach, we employ collapsed Gibbs sampling, in which we marginalize out the topic parameters $\beta$ and perdocument topic proportions $\theta_{d}$ to speed up the convergence. Therefore, the posterior we need to approximate is $p\left(\mathbf{c}_{1: D}, \mathbf{z}_{1: D} \mid \gamma, m, \pi, \eta, \mu, \tau, \mathbf{w}_{1: D}, \mathbf{y}_{1: D, 1: D}\right)$, where $\gamma$ and $\eta$ are the hyperparamters of nCRP and the topic-word distribution, $\{m, \pi\}$ is the sticking-breaking parameter for topic proportions. In this work we do not provide the parameter learning algorithm and these parameters are fixed according to the analysis and prior expectation about the data, which is discussed in the section VI. In each iteration, the Gibbs sampler is divided into two steps: 1) For each document $d$, re-sample the per-word level allocations $z_{d, n}$ and per-document paths $\mathbf{c}_{d}$ based on 
observations of $\mathbf{w}_{d}{ }^{3}$, and 2) For each pair of documents $\left(d, d^{\prime}\right)$, re-sample the per-word level allocations $z_{d, n}$ and per-document paths $\mathbf{c}_{d}$ based on observations of $y_{d, d^{\prime}}$.

1) Gibbs sampling based on $\mathbf{w}_{1: D}$ : The first step includes two parts: re-sample the per-word level allocations $z_{d, n}$ with given path assignments, and re-sample the perdocument paths $\mathbf{c}_{d}$ with given level allocations.

Sampling Level Allocations. Given the current path assignments, we need to re-sample the level allocation variable $z_{d, n}$ for word $n$ in each document $d$ :

$$
\begin{aligned}
& p\left(z_{d, n} \mid \mathbf{z}_{-(d, n)}, \mathbf{c}, \mathbf{w}, m, \pi, \eta\right) \propto \\
& p\left(w_{d, n} \mid \mathbf{z}, \mathbf{c}, \mathbf{w}_{-(d, n)}, \eta\right) p\left(z_{d, n} \mid \mathbf{z}_{d .-n}, m, \pi\right)
\end{aligned}
$$

This is the same notation with the standard hLDA [7]. ShLDA differs with it in the first term, which is the probability of a given word based on a possible assignment. In standard hLDA, it is assumed that the topic parameters $\beta$ are generated from a symmetric Dirichlet distribution:

$$
\begin{aligned}
& p\left(w_{d, n} \mid \mathbf{z}, \mathbf{c}, \mathbf{w}_{-(d, n)}, \eta\right) \propto \\
& \#\left[\mathbf{z}_{-(d, n)}=z_{d, n}, \mathbf{c}_{z_{d, n}}=c_{d, z_{d, n}}, \mathbf{w}_{-(d, n)}=w_{d, n}\right]+\eta
\end{aligned}
$$

where \#[.] counts the elements of an array satisfying a given condition. Let $q_{d, n}$ denote the left side of the above equation. We incorporate the supervision of seed word set by setting a soft constraint to modify the Gibbs sampling process that seed words tend to be generated from the root topic $\left(z_{d, n}=1\right)$ :

$$
\hat{p}\left(w_{d, n} \mid \mathbf{z}, \mathbf{c}, \mathbf{w}_{-(d, n)}, \eta\right) \propto q_{d, n} \cdot \operatorname{Constraint}\left(\mu, z_{d, n}\right)
$$

where the definition of Constraint $\left(\mu, z_{d, n}\right)$ is in Eq.3. Following this sampling process, the words relevant to the query are guaranteed to have a higher probability to be assigned the root topic, leaving the subtopics focusing more on refined terms. The second term in Eq.5 is a distribution over levels which is concerned with the GEM distribution of the stick breaking process. We keep it unchanged.

The step of sampling path assignments is identical to that in standard hLDA (see [7] for detail).

2) Gibbs sampling based on $\mathbf{y}_{1: D, 1: D}$ : Within each iteration, after re-sampling the per-word level allocations $z_{d, n}$ and per-document paths $\mathbf{c}_{d}$ based on observations of $\mathbf{w}_{d}$, the second step is to modify the posterior probabilities based on the observed duplicate links $\mathbf{y}_{1: D, 1: D}$.

For each pair of documents $\left(d, d^{\prime}\right)$, if the binary duplicate link $y_{d, d^{\prime}}=0$, we can not determine the topic similarity of the two documents and thus keep the posterior probabilities unchanged in this iteration. For path assignments $\mathbf{c}_{d}$ and $\mathbf{c}_{d^{\prime}}$, if $y_{d, d^{\prime}}=1$, the probabilities are updated as:

$$
\begin{aligned}
& p\left(\mathbf{c}_{d} \mid \mathbf{c}_{-d}, \mathbf{z}, \eta, \gamma, \mathbf{w}_{d}, \mathbf{y}_{d, d^{\prime}}\right) \\
& =0.5 *\left[p\left(\mathbf{c}_{d} \mid \mathbf{c}_{-d}, \mathbf{z}, \eta, \gamma, \mathbf{w}_{d}\right)+p\left(\mathbf{c}_{d^{\prime}} \mid \mathbf{c}_{-d^{\prime}}, \mathbf{z}, \eta, \gamma, \mathbf{w}_{d^{\prime}}\right)\right]
\end{aligned}
$$

\footnotetext{
${ }^{3}$ The first step is the Gibbs sampling for the proposed ShLDA.
}

$$
\begin{aligned}
& p\left(\mathbf{c}_{d^{\prime}} \mid \mathbf{c}_{-d^{\prime}}, \mathbf{z}, \eta, \gamma, \mathbf{w}_{d^{\prime}}, \mathbf{y}_{d, d^{\prime}}\right) \\
& =0.5 *\left[p\left(\mathbf{c}_{d} \mid \mathbf{c}_{-d}, \mathbf{z}, \eta, \gamma, \mathbf{w}_{d}\right)+p\left(\mathbf{c}_{d^{\prime}} \mid \mathbf{c}_{-d^{\prime}}, \mathbf{z}, \eta, \gamma, \mathbf{w}_{d^{\prime}}\right)\right]
\end{aligned}
$$

where on the right side is the posterior probabilities computed from the first step. In this way, we restrict the path assignments of document $d$ and $d^{\prime}$ to be the same if their duplicate link $y_{d, d^{\prime}}=1$, i.e., the subtopics of the documents are identical if they are duplicate with each other.

For level allocations $\mathbf{z}_{d, 1: N_{d}}$ and $\mathbf{z}_{d^{\prime}, 1: N_{d^{\prime}}}$, if if $y_{d, d^{\prime}}=$ 1, the probabilities are updated as:

$$
\begin{aligned}
& p\left(z_{d, n} \mid \mathbf{z}_{-(d, n)}, \mathbf{c}_{d}, m, \pi, \eta, \tau, \mathbf{w}_{d}, \mathbf{y}_{d, d^{\prime}}\right) \\
& \propto p\left(\mathbf{y}_{d, d^{\prime}} \mid \mathbf{c}_{d}, \mathbf{c}_{d^{\prime}}, \theta_{d}, \theta_{d^{\prime}}, \tau\right) \cdot p\left(z_{d, n} \mid \mathbf{z}_{-(d, n)}, \mathbf{c}_{d}, m, \pi, \eta, \mathbf{w}_{d}\right)
\end{aligned}
$$

where the first term on the right side is computed according to Eq.4, $\theta_{d}$ and $\theta_{d^{\prime}}$ are computed by the level assignments $\mathbf{z}_{d, 1: N_{d}}$ and $\mathbf{z}_{d^{\prime}, 1: N_{d^{\prime}}}$ from the step, while the second term on the right side is the posterior probabilities from the first step.

With these conditional distributions, the full Gibbs sampling process is specified. Given current state of the sampler, $\left\{\mathbf{c}_{1: D}^{(t)}, \mathbf{z}_{d, 1: D}^{(t)}\right\}$, we iteratively sample each variable conditioned on the rest:

i. For each document $d$ :

(a) Randomly draw $\mathbf{c}_{d}^{(t+1)}$ as in standard hLDA.

(b) Randomly draw $\mathbf{z}_{d, 1: N_{d}}^{(t)}$ from Eq.5.

ii. For each pair of documents $d, d^{\prime}$ :

(a) Update $\mathbf{c}_{d}^{(t+1)}$ and $\mathbf{c}_{d^{\prime}}^{(t+1)}$ from Eq.8 and Eq.9.

(b) Update $\mathbf{z}_{d, 1: N_{d}}^{(t)}$ and $\mathbf{z}_{d^{\prime}, 1: N_{d^{\prime}}}^{(t)}$ from Eq.10.

After running for sufficiently iterations, we can approach its stationary distribution, which is the conditional distribution of the latent variables in the RShLDA model given the corpus, seed word set and duplicate links.

\section{QUERY EXPANSION AND DUPliCATE DETECTION}

\section{A. Query Expansion}

Query expansion $(\mathrm{QE})$ is the process of reformulating a seed query to improve retrieval performance in information retrieval operations. In our case, we employ query expansion, combining WordNet and association mining to extend the query terms into a seed word set $\mathcal{S}$, which guide the discovery of the root topic in the derived topic hierarchy.

WordNet [19] is an online lexical dictionary which describes word relationships in three dimensions of Hypernym, Hyponym and Synonym. It is organized conceptually. According to our mechanism of incorporating the supervision information, adding noisy words not included in the vocabulary will not detract from the topic modeling process. This means we are allowed to extend the query as much as we can, on condition that no words concerned with subtopics are mixed. Therefore, we exclude words having hyponym or troponym relations to the query in 
WordNet. In addition, instead of removing unusual words, we employ association mining and add high-frequency words into the seed word set.

We utilize WordNet as the basic rule to extend the query along two dimensions including hypernym and synonym relations. Since WordNet has narrow coverage for domain specific queries [20], we use association rules to exploit collection-dependent word relationships. We examine the vocabulary and add the words with both top 10 highest con fidence and support with the original query words into the query expansion. For example, the final seed word set of query ' $9 / 11$ attack' is $\mathcal{S}=\{911$ attack Assault aggress assail fight struggle contend onslaught onset attempt operation approach event wtc world trade center terrorist terrorism 9-11\}.

\section{B. Duplicate Detection}

Near-duplicate provides strong cues for linking related videos. If two videos are near-duplicate, they can be regarded as having a must-link constraint, which indicates that they describe the same subtopic and should be grouped into the same cluster. A binary variable $y_{d, d^{\prime}}$ is employed to denote the duplicate link between each two videos $d$ and $d^{\prime}$, which is then formulated as the observed pairwise link and integrated into the topic modeling process (see section IV.C).

We utilize the algorithm proposed in [21] for duplicate detection, which is state-of-the-art method. Sampled frames are matched based on a novel local feature indexing method, which is robust to video transformations and efficient in memory usage and computation time. The spatio-temporal consistency are verified after individual frames matching and the score for video-level matching are normalized. The technical details can be referred to the paper.

The total number of videos in our collected dataset is 5,405 . We evaluate the performance of duplicate detection in the experiments. The number of duplicate videos is 689 and the duplicate rate is $12.7 \%$, which is lower than the demonstrated number [16] probably because we only analyze the top returned videos. The F-measure of duplicate detection is $75.4 \%$. We emphasize that duplicate detection can be performed when the videos are uploaded, reducing the online time cost, which is very important for search result clustering.

\section{EXPERIMENTS}

\section{A. Dataset and Evaluation Metrics}

Since the goal of this paper is to present a multimodal framework for clustering-based web video retrieval, we assess the retrieval effectiveness in a webscale video dataset collected from video sharing websites. After careful examination of the hottest topics in Youtube, Google Zeitgeist, and Twitter, we selected seven social and political topics as queries. We issued these queries to Youtube, Metacafe and Vimeo, and crawl the top 500, 150 and 150 (if there are) returned videos for experiments,

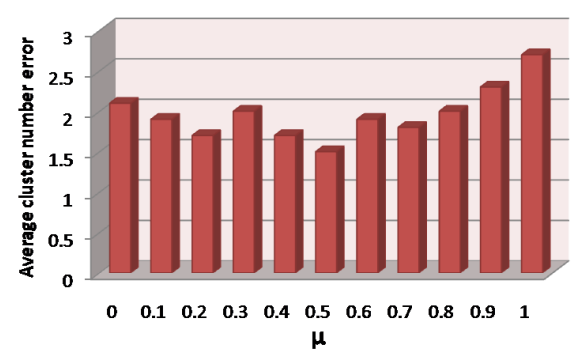

Figure 4. Average subtopic number error as $\mu$ changes.

respectively. We focused on the topmost search results to avoid bringing too many unrelated videos. Videos with no tags are filtered out. The videos collected from each query form a video set. The queries and statistics about the corresponding video set are listed in Table I.

To evaluate the performance of proposed methods, we implement BCS [5], LDA and the standard hLDA as the baseline for comparisons. As far as we know, BCS is the only existing work addressing the problem of video search result clustering. They cluster the top returned videos based on visual similarity of low-level appearance features and textual similarity of term vector features. We compare the different methods on retrieval performance and the clustering quality. For the retrieval performance, we use the metrics of subtopic reach time (SRT, [22]) and time cost. SRT is proposed in text search result clustering, which is a modelization of the time taken to locate a relevant document. For the clustering quality, we access the metrics of purity [23], F measure [24] and clustering description readability.

\section{B. Parameter Settings}

Topic models make assumptions about the topic structure by the settings of hyperparameters. We empirically fixed the hyperparameters according to the prior expectation about the data. The hyperparameter $\eta$ controls the smoothing/sparsity of topic-word distribution. Small $\eta$ encourages more words to have high probability in each topic. (For LDA, it requires less topics to explain the data. For hLDA, ShLDA and RShLDA, it leads to a small tree with compact topics.) Delighted from this, we empirically chose a relatively small value of $\eta$ and set $\eta=0.5$. All the hierarchical topic models have an additional hyperparameter, CRF parameter $\gamma$, which decides the size of the inferred tree. As in [25], we set $\gamma=1$ to reduce the likelihood choosing new paths when traversing nCRP.

We then discuss the choice of supervision strength parameter $\mu$ in ShLDA and the coefficient parameter $\tau$ in RShLDA. We utilize a benchmark text subtopic retrieval dataset, AMBIENT $^{4}$, for the determination of $\mu$. We assume that appropriate $\mu$ brings no perturbation to the hierarchical topic discovery process and the derived topic tree should be consistent with the latent hierarchical structure. Therefore, we analyzed the error between the

\footnotetext{
${ }^{4}$ http://credo.fub.it/ambient
} 
TABLE I.

COLlECTED WEB VIDEO DATASET STATISTICS

\begin{tabular}{cccccc}
\hline ID & Query & \#Video retrieved & \#Video collected & \#Vocabulary & \#Words \\
\hline 1 & $9 / 11$ attack & 8,361 & 791 & 2140 & 38747 \\
\hline 2 & gay rights & 602,885 & 799 & 2048 & 35538 \\
\hline 3 & abortion & 66,606 & 797 & 1770 & 33144 \\
\hline 4 & Iraq war invasion & 4,425 & 702 & 1778 & 36760 \\
\hline 5 & Beijing Olympics & 202,511 & 787 & 1718 & 32370 \\
\hline 6 & Israel palestine conflict & 252,746 & 798 & 1814 & 38499 \\
\hline 7 & US president election & 36,037 & 731 & 1792 & 33249 \\
\hline
\end{tabular}



Figure 5. The ground-truth subtopic number and automatically derived cluster number for the test queries

subtopic number of ground truth and the derived subtopic number of ShLDA over the different values of $\mu$ (see Fig.4). $\mu=0.5$ achieves the least error. Therefore, we fixed $\mu=0.5$ in following experiments. As we build a two-level topic hierarchy and all the videos share the same root topic, $\tau$ is a two-dimensional vector and $\tau_{1}$ can be set to $0 . \tau_{2}$ is empirically set as 0.1 .

The most important parameters for BCS are the weights for adopted features, visual, tag, title and description. Affinity propagation (AP) and normalized cut (NC) are utilized as the clustering algorithm and they demonstrated AP generally outperforms NC. Therefore, we fixed the set of feature weights showing best performance with AP clustering: visual-0.3, tag-0.49, title- 0.07 , description0.14 .

Furthermore, we notice that, AP-based BCS, hLDA and the proposed ShLDA and RShLDA can automatically determine the number of clusters, which is reasonable in practical implications. However, LDA must be given the number of clusters as input. Choosing the appropriate number of clusters is challenging, especially for postretrieval clustering task. To simplify the experimental configurations, we fix the cluster number as the ground truth value for LDA.

\section{Experimental Results}

1) Visualization of the discovered subtopics: We visualize the discovered subtopics of video collections for the seven test queries in Fig.11 and Fig.12. For the query of '9/11 attack', the subtopics derived from LDA and topic hierarchies derived from hLDA, ShLDA and RShLDA are presented in Fig. 11 for comparison. It is shown that LDA mixes common words like 'attack', '911', 'September', 'terrorist' in different subtopics and fails to discover the shared topic. Compared with flat structure based clustering method of LDA, utilizing the hierarchical topic models will prevent the shared topic

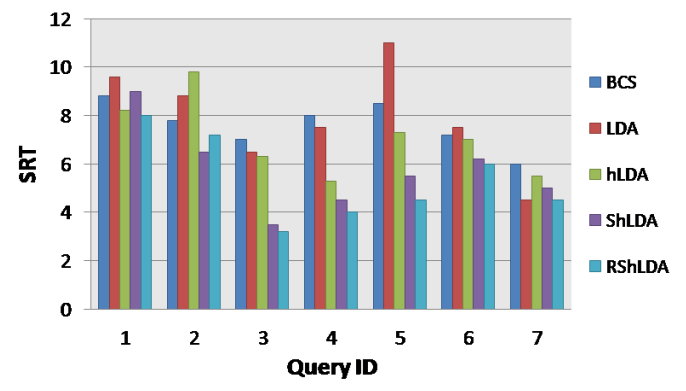

Figure 6. SRT of different clustering methods

from being mixed within other topics and thus ensure the clustering performance. The topic hierarchy recovered by hLDA finds the shared topic on the root level. However, without constraint of topic distribution over the seed word set, words describing the shared topic, e.g. 'wtc', 'terrorist', '11', 'attack' also appear in subtopics. This contaminates the subtopics and limits its power to sub-events or viewpoints detection. Incorporated with supervision information, ShLDA prevents seed words generating from the subtopics, and results in a topic hierarchy with subtopics focusing on the refined themes. RShLDA further improves over ShLDA by considering the visual cues. With the aid of duplicate constraints, the visual similar videos are guaranteed to be clustered together and RShLDA uses link information to influence the topic hierarchy and the subtopic distribution.

2) Comparing different clustering methods: For evaluation, human accessors create ground-truth subtopic themes after browsing the retrieved videos for each query-corresponding video set. For example, the subtopic themes inside the video collection derived from the query 'abortion' are summarized as pro-abortion, anti-abortion and neutral. Videos are manually labeled as belonging to a certain subtopic (cluster). The ground-truth subtopic number and derived subtopic (cluster) number by BCS, hLDA, ShLDA and RShLDA for the test queries are shown in Fig.5. We can see that all four models fail to recover the ground-truth subtopic number for some video sets. The reason is that the ground-truth subtopic themes created by subjective assessment may not reflect the nature of the video set, especially when unrelated noisy videos are involved. We also notice that RShLDA, ShLDA and hLDA performs better than BCS. The BCS curve is high above the ground truth. This is due to its duplicate clustering alike mechanism, which results in small-size duplicate video clusters. 


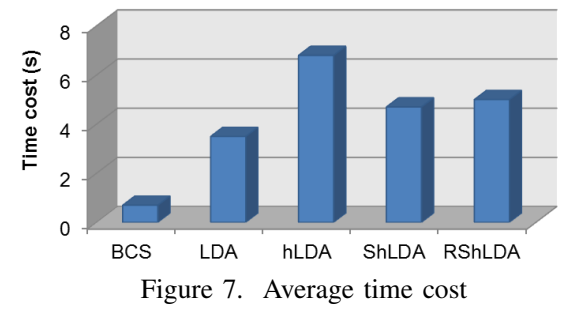

We first compare the SRT and time cost of different clustering methods (see Fig.6 and Fig.7). Note that BCS and RShLDA utilize additional visual information. From Fig.6, we can see the best performance is achieved by RShLDA, followed by ShLDA and hLDA, which is due to the multi-modal framework and the separation of shared common topic from subtopics. It is interesting to see that the BCS basically superiors over LDA, and achieves even better SRT than ShLDA for query 1. The reason is that BCS additionally utilizes visual information for clustering. However, BCS suffers from two problems: 1) it employs a flat-structure clustering algorithm; 2) it uses the cluster centroid to represent the cluster and provides no mechanism for how to derive the cluster labels.

For clustering-based video retrieval, the clustering is performed online, which requires necessarily short response time. We focus on the efficiency of clustering algorithms and do not consider about the video acquisition time cost. We assume that visual features used in BCS are extracted offline and take no account of text preprocessing time. Fig.7 illustrates time complexity for the clustering algorithms. For ShLDA and RShLDA we do not consider the query expansion time, and the query expansion time from local-stored WordNet is about 1.4s. As duplicate detection an be performed when videos are uploaded, we also exclude the time cost of duplicate detection for RShLDA. Since BCS uses AP for clustering, it achieves lower time cost than the generative topic models. The speedup of ShLDA over hLDA is due to that incorporated prior guides the seed words gradually generated from the root set and thus speedups the convergence process. RShLDA costs a little more training time than ShLDA as the available positive duplicate links are very small compared to the huge pairwise link number.

We noticed that the computational cost dramatically increases when dealing with large-scale web videos, and we will be researching towards this in future work. In addition to develop faster hierarchical topic models, we can design a composite UI to improve user experience while waiting for the cluster-based results: The search engine returns the conventional list-based results as soon as users issue the queries. The server conducts topic modeling while users browse through the list-based results. Once the training is finished, the videos will be mapped to the corresponding subtopics and cluster-based results will be presented to the users. Instead of online training, we can also perform offline training for all pre-chosen queries on the server. Two schemes are considered for offline training. The first is re-training models for all the queries at intervals on the server, when bunches of new videos are uploaded.
The second is estimating the topic (related queries) of the uploaded videos, and developing algorithms for model update and modifying topic models for related queries.

Besides accessing the retrieval performance, we also evaluate the clustering qualities. Cluster purity is used to evaluate the performance of video clustering:

$$
\text { purity }=\frac{1}{N} \sum_{i=1}^{N} \frac{\max _{j}\left|C_{i} \cap S_{j}\right|}{\left|C_{i}\right|}
$$

where $N$ is the number of videos in the collection, $C_{i}$ is the set of videos in the $i^{t h}$ cluster and $S_{j}$ is the set of videos in the $j^{\text {th }}$ subtopic. Fig.8 shows the cluster purity. We find that BCS noticeably outperforms the other algorithms. High purity is easy to achieve when the number of clusters is large. Therefore, we cannot use purity to trade off the quality of the clustering against the number of the clusters. A measure to make this tradeoff is $F$ measure. We evenly penalize false negatives and false positives, i.e. the F1 measure (see Fig.9). It is shown that BCS performs poorly on F1 measure, even much worse than LDA. The reason is that BCS focuses on clustering duplicate or near-duplicate videos, which limits the cluster size and forces considerable number of semantically similar videos assigned to different clusters.

The quality of the cluster description is crucial to the usability of clustering-based video retrieval. If a cluster cannot be described, it is presumably of no value to the user. BCS employs the cluster centroid as the cluster representation, which lacks real descriptions and is of litter use for guiding the user understanding the cluster content. The cluster description readability is evaluated as follows. Each subtopic characterized by the top five probable words was shown to the participants with the top three ranked videos within this subtopic. The participants were asked to evaluate the cluster description readability in two aspects: 'whether the topic description itself is sensible, comprehensive and compact' (question 1) and 'whether the topic description is consistent with the representative videos' (question 2). For each question, participants rated from one to five where five is best. The average ratings are shown in Fig.10. The proposed RShLDA and ShLDA show superiority on generating meaningful cluster descriptions, especially on generating sensible, comprehensive and compact representations (question 1). We note that ratings for query 5 - 'Beijing Olympics' are relatively low. In the retrieved video set of 'Beijing Olympics', diverse events or subtopics are involved, e.g. opening ceremony, game video, athlete interview, torch relay, etc. The discovered topic structure is sparse and less meaningful. Besides, some unrelated videos regarding issue of Tibet are also included.

\section{CONCLUSIONS}

In this paper, we have presented a hierarchical topic model based multi-modal framework for web video faceted subtopic retrieval. Instead of showing a long ranked list videos, we explore the hierarchical topic 


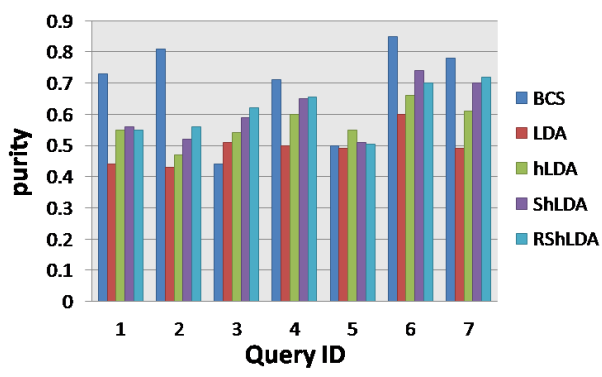

Figure 8. Purity rates

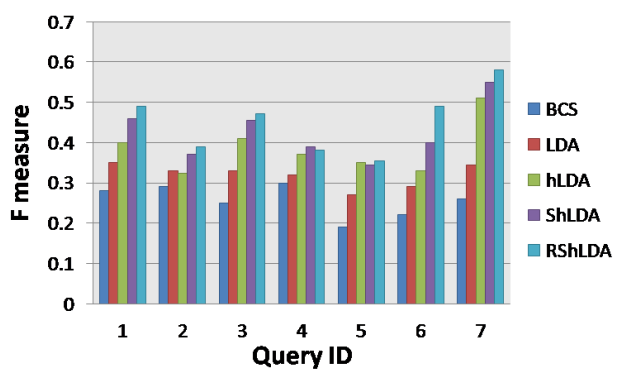

Figure 9. F measure for different methods.

structure in the retrieved video collection returned from faceted queries and present users with videos organized by semantic clusters. Experiments demonstrate the effectiveness of the proposed method.

In the future, we will improve our current work along the following directions. 1) Unrelated videos in retrieved video collections will affect the clustering performance. We will develop noisy subtopic aware hierarchical topic model to reduce the influence of noises as well as remove unrelated videos. 2) In this paper, we do not provide the algorithm of learning the hyper parameters. Posterior inference and parameter estimation are two basic computation issues for nonparameteric Bayes modeling. Designing the parameter estimation algorithm is one of our future work.

\section{ACKNOWLEDGEMENT}

This work was in part supported by National Natural Science Foundation of China (Grant No. 90920303) and National Program on Key Basic Basic Research Project (the 973 Program, Project No. 2012CB316304).

\section{REFERENCES}

[1] S. Osinski and D. Weiss, "A concept-driven algorithm for clustering search results," Intelligent Systems, IEEE [see also IEEE Intelligent Systems and Their Applications], vol. 20, no. 3, pp. 48-54, 2005.

[2] H.-J. Zeng, Q.-C. He, Z. Chen, W.-Y. Ma, and J. Ma, "Learning to cluster web search results," in SIGIR, 2004, pp. 210-217.

[3] D. Cai, X. He, Z. Li, W. Y. Ma, and J. R. Wen, "Hierarchical clustering of www image search results using visual textual and link information," in ACM Multimedia, 2004, pp. 952-959.

[4] F. Jing, C. Wang, Y. Yao, K. Deng, L. Zhang, and W. Y. Ma, "Igroup: web image search results clustering," in $A C M$ Multimedia, 2006, pp. 377-384.

[5] A. Hindle, J. Shao, D. Lin, J. Lu, and R. Zhang, "Clustering web video search results based on integration of multiple features," World Wide Web, vol. 14, no. 1, pp. 1-21, 2010.
[6] Z. M. Zhang, Z. Zhang, R. Jain, and Y. Zhuang, "Overview of acm international workshop on connected multimedia," in Proceedings of the international conference on Multimedia, 2010, pp. 1763-1764.

[7] D. M. Blei, T. L. Griffiths, and M. I. Joradan, "The nested chinese restaurant process and bayesian nonparametric inference of topic hierarchies," J. ACM, vol. 57, no. 2, pp. $1-30,2010$.

[8] B. Carterette and P. Chandar, "Probabilistic models of ranking novel documents for faceted topic retrieval," in Proceeding of the 18th ACM conference on Information and knowledge management, 2009, pp. 1287-1296.

[9] C. L. Clarke, N. Craswell, and I. Soboroff, "Overview of the trec 2009 web track," Tech. Rep.

[10] W.-H. Lin and A. G. Hauptmann, "News video classification using svm-based multimodal classifiers and combination strategies," in ACM Multimedia, 2002, pp. 323-326.

[11] L. Liu, Y. Rui, L.-F. Sun, B. Yang, J. Zhang, and S.-Q. Yang, "Topic mining on web-shared videos," in ICASSP, 2008, pp. 2145-2148.

[12] C. Ramachandran, R. Malik, X. Jin, J. Gao, and J. Han, "Videomule: a consensus learning approach to multi-label classification from noisy user-generated videos," in $M M$, 2009.

[13] J.-Y. Pan, H.-J. Yang, and C. Faloutsos, "Mmss: Multimodal story-oriented video summarization," in ICDM, 2004, pp. 491-494.

[14] D. qing Zhang, C. yung Lin, S. fu Chang, and J. R. Smith, "Semantic video clustering across sources using bipartite spectral clustering," in Spectral Clustering, International Conference on Multimedia and Expo, 2004, pp. 117-120.

[15] M. Guillaumin, J. J. Verbeek, and C. Schmid, "Multimodal semi-supervised learning for image classification," in CVPR, 2010, pp. 902-909.

[16] X. Wu, A. G. Hauptmann, and C.-W. Ngo, "Practical elimination of near-duplicates from web video search," in ACM MM, 2007, pp. 218-227.

[17] D. Blei, A. Ng, and M. Jordan, "Latent dirichlet allocation," Journal of Machine Learning Research, vol. 7, pp. 993-1022, 2003.

[18] Y. W. Teh, M. I. Jordan, M. J. Beal, and D. M. Blei, "Hierarchical dirichlet processes," Journal of the American Statistical Association, vol. 101, no. 476, pp. 1566-1581, 2006.

[19] G. A. Miller, R. Beckwith, C. Felbaum, D. Gross, and K. Miller, Introduction to WordNet: An On-line Lexical Database. Oxford Univ Press, 1990, vol. 3, no. 4.

[20] K. Chandramouli, T. Kliegr, J. Nemrava, V. Svatek, and E. Izquierdo, "Query refinement and user relevance feedback for contextualized image retrieval," in Visual Information Engineering, Xian China, 2008, pp. 452-458.

[21] M. Douze, H. Jegou, and C. Schmid, "An image-based approach to video copy detection with spatio-temporal post-filtering," IEEE Transactions on Multimedia, vol. 12, no. 4, pp. 257-266, 2010.

[22] C. Carpineto, S. Osinski, G. Romano, and D. Weiss, "A survey of web clustering engines," ACM Comput. Surv., vol. 41, no. 3, pp. 1-38, 2009.

[23] P. Tan, M. Steinbach, and V. Kumar, Introduction to Data Mining. Addison Wesley, 2005, vol. 19, no. 1.

[24] M. Steinbach, G. Karypis, and V. Kumar, "A comparison of document clustering techniques," in ACM SIGKDD, 2000, pp. $35-42$.

[25] D. M. Blei, T. L. Griffiths, M. I. Joradan, and J. Tenenbaum, "Hierarchical topic models and the nested chinese restaurant process," in Advances in Neural Information Processing Systems. MIT Press, 2004, pp. 17-24. 



Figure 10. Mean ratings of cluster description readability for (left:) Question 1 (right:) Question 2.

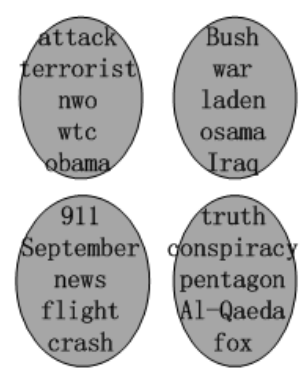

LDA



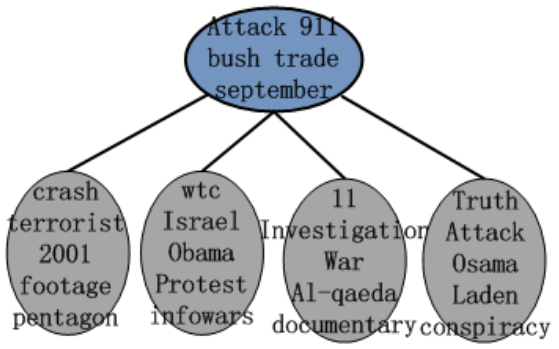

hLDA

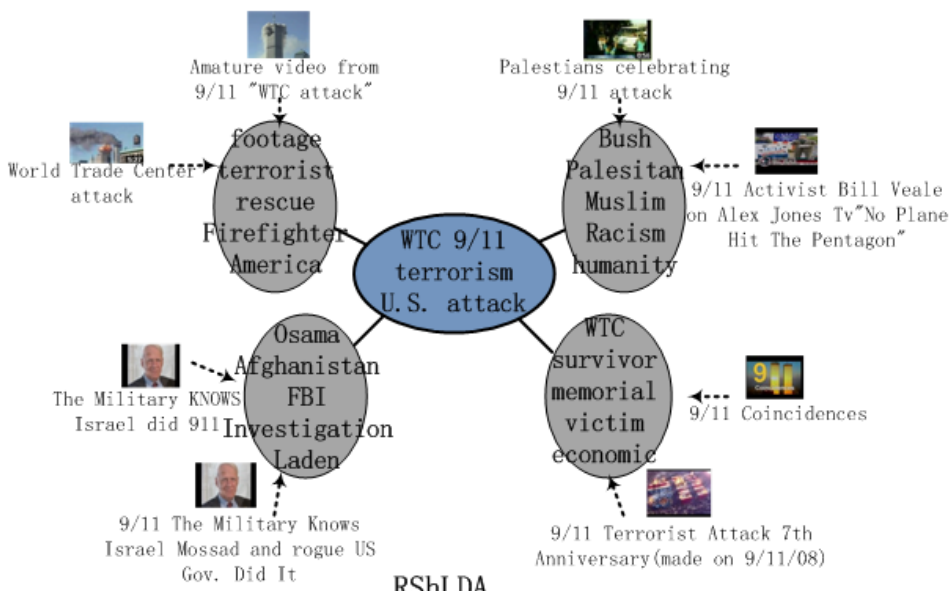

Figure 11. Discovered topic hierarchy from the video collection of query of ' $9 / 11$ attack' using LDA, hLDA, ShLDA and RShLDA. For ShLDA and RShLDA, we also present two videos having the largest proportion associated with the subtopics 




(a)

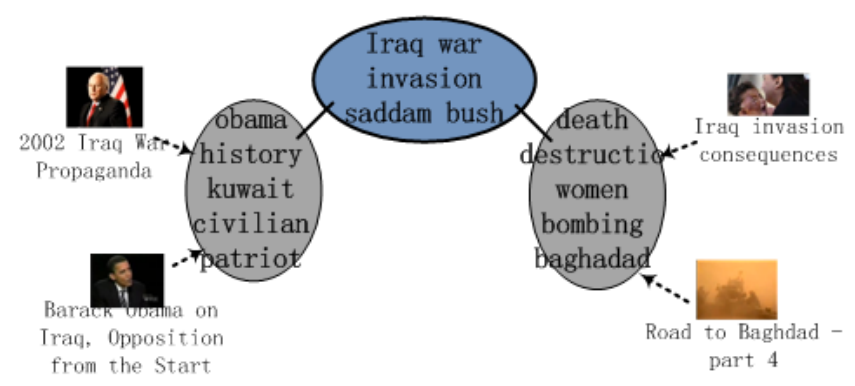

(c)

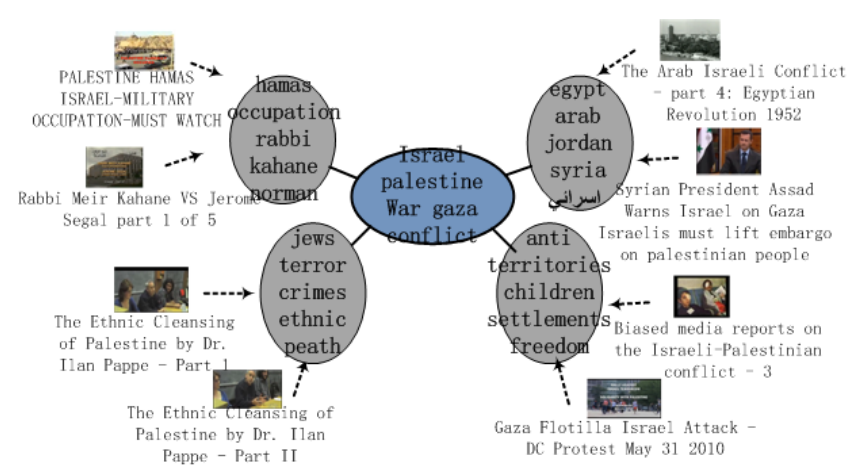

(e)

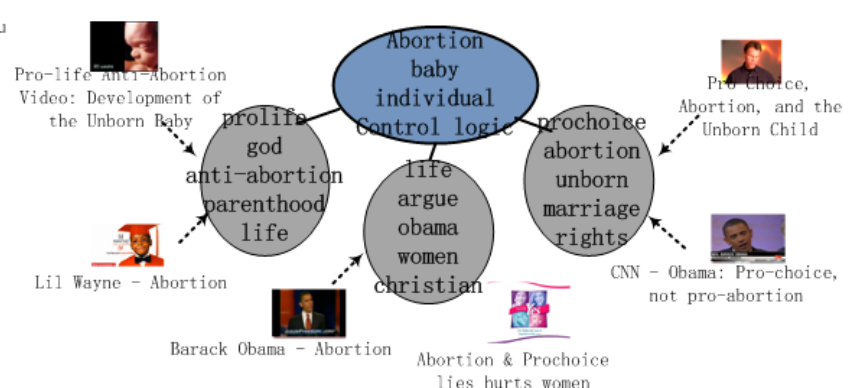

(b)

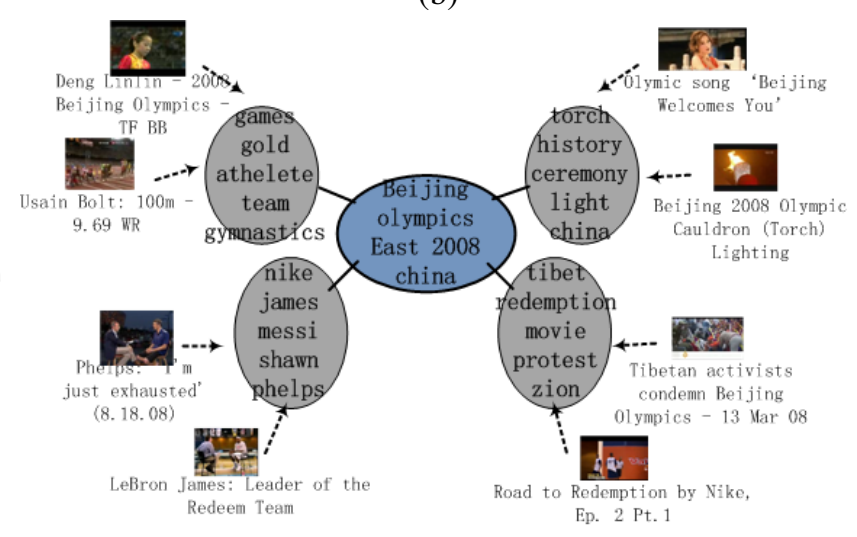

(d)

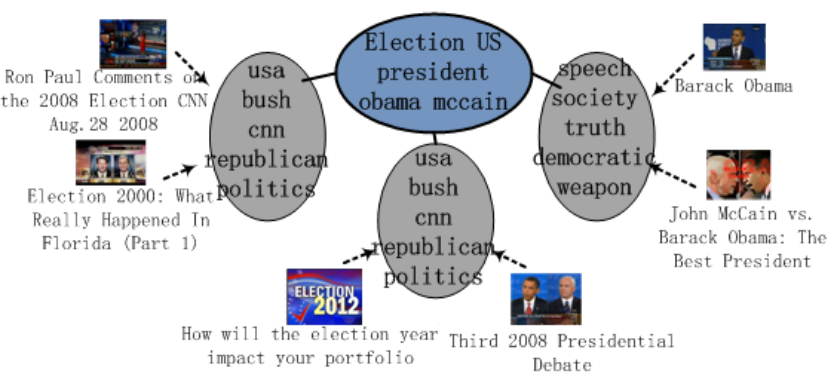

(f)

Figure 12. Discovered topic hierarchies from the video collection of queries from Youtube using RShLDA. (a) 'gay rights' (b) 'abortion' (c) 'Iraq war invasion' (d) 'Beijing Olympics' (e) 'Israeli Palestine conflicts' (f) 'US president election'. 